\title{
On Unbounded Growth with Heterogenous Consumers *
}

\author{
Martin Kaae Jensen \\ University of Birmingham, Department of Economics, 90 Vincent Drive, \\ Birmingham B15 2TT, United Kingdom.
}

\begin{abstract}
This paper studies unbounded growth with infinite-horizon consumers whose discount factors and instant utility functions are not assumed to be identical. The Negishi-type planner becomes non-homogenous and as a consequence, different consumer types may end up holding all wealth in the far future for different initial distributions of income. The main technical contribution is a turnpike theorem which generalizes the global stability results of Kaganovich (1998) and Jensen (2003).
\end{abstract}

Key words:

Unbounded Growth, Heterogenous Consumers, Ramsey Conjecture, Balanced Growth Equilibrium, Unbalanced Growth, Turnpike, Global Stability.

JEL-classification:

D51, D90, C61, C62.

\section{Introduction}

This paper studies heterogenous consumers in a framework with unbounded growth. A general time-stationary technology set whose output is not restricted by decreasing returns, is paired with a population of infinite-horizon consumers whose discount factors and instant utility functions are not assumed to be identical. A representative agent does not exist and equilibria

ऋ I would like to thank Birgit Grodal, Herakles Polemarchakis, Manuel Santos, Karl Vind, and an anonymous referee for many useful comments and suggestions. Responsibility for remaining errors rests with me. The first version of this paper was written while the author was visiting Brown University in 2003-2004.

Email address: m.k.jensen@bham.ac.uk (Martin Kaae Jensen).

URL: http://www.economics.bham.ac.uk/jensen (Martin Kaae Jensen).

Preprint submitted to Elsevier Science 5 February 2006 
will therefore depend on the initial distribution of income, differences in savings behavior, and other types of household heterogeneity.

Within this framework, the paper studies steady states (balanced growth equilibria) as well as the stability properties of these. The results may broadly be divided into two groups: One concerns the global stability or turnpike properties of such balanced growth equilibria (BGEs). The other is concerned with the connection between multiplicity of steady states and heterogeneity. From a technical perspective, the two groups turn out to be very closely related as we now attempt to explain.

Within the paper's framework, a Negishi-type social planner (cf. Negishi (1960), Bewley (1982)) will not correspond to a homogenous program except in very special cases. Thus the model departs almost immediately from standard unbounded growth models as investigated by Alvarez and Stokey (1998), Kaganovich (1998), Jensen (2003), and many others. ${ }^{1}$ The model also departs from traditional growth theory in the more obvious sense that steady states are not stationary but expansive. It can however be seen as an integration of the two, i.e., of unbounded growth models and traditional intertemporal equilibrium models in the tradition of Bewley (1982). Now, in the latter which from now on will be referred to as bounded growth models - the role of consumer heterogeneity is well understood. One of its key properties is the so-called Ramsey-conjecture: As time goes to infinity, a Negishi-type social planner will place zero weight on any consumer whose discount factor is below the highest discount factor in the population (Becker (1980), Boyd (2000)). Thus one can identify a group of consumers who will eventually come to dominate the economy in the sense that equilibria will approach those of a limit economy consisting of these consumers alone. One implication of this is that the set of steady states can be characterized independently of the initial distribution of income (Coles (1985), Yano (1998)). From a practical perspective there is then no loss of generality in studying a reduced model featuring only the consumers with the highest discount factor. Such a reduced economy give rise to an additive and stationary social planner problem thus paving the way for a frictionless integration with turnpike theory (Bewley (1982)).

As it turns out, none of the above is true in a setting with unbounded growth. As we shall argue, the method by which one "reduces to agents with the same savings behavior" becomes fallible, if not flawed. Balanced growth equilibria depend explicitly on the long-run income distribution which in turn is a function of the interest rate. The interest rate is, however, endogenous and depends on the initial distribution of income. ${ }^{2}$ In section 3 we shall exem-

$\overline{1}$ In the applied literature such models have found very wide applications. An example is in the debate about taxes' influence on economic growth (cf. Stokey and Rebelo (1995) and references therein).

2 This is in contrast to bounded growth models where the interest factor becomes 
plify these observations by considering an economy where a redistribution of income at date 0 permanently effects the long-run income distribution, and hence also the interest and growth rates. ${ }^{3}$ By implication, there is no way to reduce such an economy to a homogenous consumer model without a considerable loss of generality: Even if attention is restricted to steady states; different steady states may be associated with different subsets of the consumers, hence if one reduces to a homogenous program one implicitly chooses a steady state and this steady state might never have been reached depending on the initial distribution of income.

Given the observations in the previous paragraph, it is clear that when in section 4 we investigate global stability, we must do so in all generality, i.e., without arbitrarily reducing to a homogenous program. The key here is that if we assume that some group of consumers becomes sufficiently patient relative to the rest (which is the usual turnpike condition, see Coles (1985)); then in fact homogenous programming does apply as a good approximation. This then brings us full circle with the main difference from the bounded growth model being that there the most patient consumers will become dominant as time passes quite independently of how patient this group is compared to everyone else.

\section{The Basic Set-up and Some First Results}

This section integrates the, by now text-book dynamic equilibrium model of Bewley (1982), Coles (1985), and others, with competitive unbounded growth theory. The literature's ruling assumption about the existence of a representative agent will be dismissed, leaving the framework of homogenous programming (Alvarez and Stokey (1998)) in favor of heterogenous consumer equilibrium theory in the spirit of Arrow and Debreu.

equal to the inverse of the highest discount factor as time approaches infinity.

3 The main point is not, however, that there are multiple balanced growth equilibria with different growth rates. That this can occur in unbounded growth models is well known (see e.g., Ladron-de-Guevara, Ortigueira and Santos (1999)). The main point is the first sentence after this footnote. Nor should this be confused with the observation made by Boyd (2000) that it is misleading to identify "patience" with the discount factor in the unbounded growth model. It might still be the case that in the long run, the initial distribution of income has no effect on equilibria. Thus in the one-good linear technology case studied by Boyd (2000): Although which consumer group ends up dominating the economy depends on the technology; the technology uniquely determines this group. In particular the initial distribution of income is of no relevance from a long-run perspective and just as in the bounded model one may as well consider an economy consisting only of a single consumer. 
Time is discrete, indexed $t$, and runs from date 0 into infinity. There are $N \in \mathbf{N}$ goods at every date, and $N^{c} \leq N$ of these are consumption goods. All goods are reproducible. The agents are $I \in \mathbf{N}$ infinitely lived consumers and an aggregate firm with constant returns to scale. A consumption plan for consumer $i \in \mathcal{I}=\{1, \ldots, I\}$ is denoted $\mathbf{x}^{i}=\left(x_{t}^{i}\right)_{t=0}^{\infty} \cdot \mathbf{y}=\left(y_{t}\right)_{t=0}^{\infty}$ denotes a production plan. Finally, $\mathbf{p}=\left(p_{t}\right)_{t=0}^{\infty}, p_{t} \in \mathbf{R}_{++}^{N}$, denotes a price sequence, and $p_{t}^{c} \in \mathbf{R}_{+}^{N_{c}}$ the first $N_{c}$ coordinates of $p_{t}$, which are indexed so as to correspond to the consumption price vector. Consumers are assumed to have preferences over the same subset of goods, $\left\{1, \ldots, N^{c}\right\}$, but this is of no importance for the results.

\section{Production}

A feasible production plan is a sequence $\mathbf{y}=\left(y_{t}\right)_{t=0}^{\infty}$, where $y_{t}=\left(-k_{t-1}, z_{t}\right)$ $\in Y \subset \mathbf{R}_{-}^{N} \times \mathbf{R}_{+}^{N}$, all $t$. $Y$, the production set, describes how input vectors at date $t-1, k_{t-1}$, can be transformed into net-output, $z_{t}$, at date $t$. At date zero, the firm has a given endowment of outputs $z_{0} \gg 0$, which can be thought of as produced by predetermined inputs at $t=-1 .^{4}$

Consider a point $(-k, z) \in \partial Y$, where $\partial Y$ denotes the set of points at the boundary of $Y$. Denote the normal cone to $Y$ at $(-k, z)$ by $N_{(-k, z)} Y{ }^{5}$ If $Y$ is convex, the normal cone is non-empty for all points at the boundary. Let $P(Y)=\cup_{(-k, z) \in \partial Y} N_{(-k, z)} Y$, i.e., the set of vectors which generate supporting hyperplanes for $Y$. Finally, let $\tilde{P}(Y)=P(Y) \cap S_{+}^{2 N-1}$, where $S_{+}^{2 N-1}=\{(p, q) \in$ $\left.\mathbf{R}_{+}^{N} \times \mathbf{R}_{+}^{N}:\|(p, q)\|=1\right\}\left(S_{++}^{2 N-1}=S^{2 N-1} \cap \mathbf{R}_{++}^{2 N}\right)$. The following assumption is standard.

Assumption $1 Y$ is a closed, convex cone with vertex zero. $(0, z) \in Y$ implies that $z=0$; there exists $(-k, z) \in Y$ with $z \gg 0$, and if $(-k, z) \in Y$, then $\left(-k^{\prime}, z^{\prime}\right) \in Y$ whenever $(-k, z) \geq\left(-k^{\prime}, z^{\prime}\right)$. Finally, $\tilde{P}(Y)$ is a closed subset of $S_{++}^{2 N-1}$.

Given a sequence of prices $\mathbf{p}=\left(p_{t}\right)_{t=0}^{\infty}$, the firm's set of maximizing production plans is given by the supply correspondence:

$$
\tilde{\mathbf{y}}(\mathbf{p})=\arg \max \left\{\sum_{t=0}^{\infty} p_{t}\left(z_{t}-k_{t}\right):\left(-k_{t-1}, z_{t}\right) \in Y \text { all } t \geq 1, z_{0} \gg 0 \text { given }\right\}(1)
$$

Note that since $Y$ is a cone, the supply correspondence is only well-defined for

$\overline{4}$ Let $x, y \in \mathbf{R}^{N} . x>y$ means that $x \geq y, x \neq y . x \gg y$ means that $x^{n}>y^{n}$, all $n=1, \ldots, N$.

5 The normal cone to a point $x \in X \subset \mathbf{R}^{N}$ is defined as $N_{x} X=\{q \in \mathbf{R}: q x \geq$ $q y$ for all $y \in X\}$. 
those price sequences which yield finite maximum profit. Moreover, maximum profit at prices $\mathbf{p}$ must equal $p_{0} z_{0}$ since firms cannot earn strictly positive profit at any date.

\section{Consumption}

Consumers are represented by utility functions of the discounted type,

$$
U^{i}\left(\mathbf{x}^{i}\right)=\sum_{t=0}^{\infty} \delta_{i}^{t} u^{i}\left(x_{t}^{i}\right)
$$

where $u^{i}: X \rightarrow \mathbf{R}$ is the instant utility function, $X=\mathbf{R}_{+}^{N_{c}}$ the consumption set at all $t$, and $\delta_{i} \in \mathbf{R}_{++}$the discount factor. Consumer $i$ holds an ownership share in the firm $\theta^{i} \in[0,1]$, and the consumers together hold claims to the firm's entire profit so $\theta=\left(\theta^{1}, \ldots, \theta^{I}\right) \in \mathbf{S}_{+}^{I-1} \equiv\left\{x \in \mathbf{R}_{+}^{I}: \sum_{n} x_{n}=1\right\}$. Let $\pi(\mathbf{p})$ denote maximum profit obtained at prices $\mathbf{p}$. It then follows that the income of consumer $i$ is: $W^{i}(\mathbf{p})=\theta^{i} \pi(\mathbf{p})=\theta^{i} p_{0} z_{0}$. Note that $\theta$ can be interpreted as the initial distribution of income. ${ }^{6}$ Utility maximization leads to the demand correspondence:

$$
\tilde{\mathbf{x}}^{i}(\mathbf{p})=\arg \max \left\{\sum_{t=0}^{\infty} \delta_{i}^{t} u^{i}\left(x_{t}^{i}\right): \sum_{t=0}^{\infty} p_{t}^{c} x_{t}^{i} \leq W^{i}(\mathbf{p}) \text { and } x_{t}^{i} \in X \text { all } t\right\}
$$

Again, this is not necessarily well-defined at this point: Even if $W^{i}(\mathbf{p})<+\infty$, utility may become infinite, and the usual maximization criterion (based on an underlying preference relation that does not order consumption plans with infinite utility) will be employed throughout.

Let $\chi_{n} \in \mathbf{R}_{+}^{N_{c}}$ denote the (unit) vector with 1 on the $n$ 'th row and 0 everywhere else.

Assumption $2 u^{i} \in C^{2}\left(\mathbf{R}_{++}^{N_{c}}, \mathbf{R}\right), i \in \mathcal{I}$, is strictly concave and increasing $\left(D u^{i}(x) \gg 0\right.$, all $x \in$ int $\left.X\right)$. Furthermore, if $x^{n}=0, x^{m}>0$ for all $m \neq n$, then $\left\|D_{x} u^{i}\left(x+\chi_{n} \epsilon\right)\right\| \rightarrow \infty$ as $\epsilon \rightarrow 0, n \in\left\{1, \ldots, N_{c}\right\}$.

Assumption 3 For every $i \in \mathcal{I}$, there exits a positive, affine transformation of $u^{i}$ which is homogenous of degree $\alpha_{i}<1$.

6 That the distribution of income is implicitly assumed to be proportional is a convenient assumption which is of no importance for the results. In stead we could let each consumer receive part of the initial stock $z_{0}^{i}, \sum_{i} z_{0}^{i}=z_{0}$, and then calculate the distribution of income backwards from $\theta^{i}=\frac{p_{0} z_{0}^{i}}{p_{0} z_{0}}$. 
Assumption 2 is again a standard assumption in the growth literature (cf. Bewley (1982)). Assumption 3 is standard in the unbounded growth literature and is made to ensure the existence of a suitable generalization of balanced growth equilibria as discussed further in the next section.

The preference relation underlying the utility function $U^{i}(\mathbf{x})=\sum_{t=0}^{\infty} \delta^{t} u^{i}\left(x_{t}\right)$ is invariant to positive, affine transformations of $u^{i}$. Hence, under assumption 1 , there is no loss of generality in assuming that $u^{i}$ is homogenous of degree $\alpha_{i}<1$. From now on this is therefore taken to be the case. Moreover, we adopt the (abusive) terminology of saying that $u^{i}$ is homogenous of degree 0 if and only if it is log-linear: $u^{i}(x)=\sum_{n=1}^{N_{c}} \beta_{n}^{i} \log \left(x_{n}\right)$.

Equilibrium and the Social Planner Representation

Economies are parameterized by the initial stock, $z_{0} \in \mathbf{R}_{++}^{N}$, so an economy is a list $\mathcal{E}\left(z_{0}\right)=\left(\left(\delta_{i}, u^{i}, \theta^{i}\right)_{i=1}^{I}, Y ; z_{0}\right)$.

Definition $1\left(\mathbf{p},\left(\mathbf{x}^{i}\right)_{i \in \mathcal{I}}, \mathbf{y}\right)$ is a competitive equilibrium for $\mathcal{E}\left(z_{0}\right)$ if

(1) $\mathbf{x}^{i} \in \tilde{\mathbf{x}}^{i}\left(\mathbf{p}, W^{i}\right)$ all $i \in \mathcal{I}$, where $W^{i}=\theta^{i} \pi(\mathbf{p})$.

(2) $\mathbf{y} \in \tilde{\mathbf{y}}(\mathbf{p})$.

(3) $\sum_{i=1}^{I} x_{0}^{i}=z_{0}-k_{0}$ and $\sum_{i=1}^{I} x_{t}^{i}=z_{t}-k_{t}, t=1,2, \ldots$

The definition of a competitive equilibrium is completely analogous to the traditional intertemporal general equilibrium model's definition (Bewley (1982), Yano (1998)). The main difference is that the present economy's set of feasible consumption plans is not assumed to be uniformly bounded over time. Moreover, primary resources do not enter explicitly in the above. ${ }^{7}$ As in the traditional case one can use the Negishi-approach (Negishi (1960), Bewley (1982)) to study equilibria as solutions to an optimal growth problem. Thus let an equilibrium price sequence, $\mathbf{p}$, be given. By assumption 2, the Euler conditions,

$$
\delta_{i}^{t} D u^{i}\left(x_{t}^{i}\right)=\eta_{i} p_{t}^{c}, t=0,1,2, \ldots
$$

must hold for consumer $i=1, \ldots, I . \eta=\left(\eta_{1}, \ldots, \eta_{I}\right)$, is the vector of marginal utilities of income (which is uniquely determined in an equilibrium). From $\eta$

$\overline{7}$ That primary resources do not enter explicitly is a simplifying way of capturing the central new growth postulate that no resource effectively bounds production. For example labor enters production in the form of human capital which is subject to accumulation without bounds. For more on this point see e.g. Dolmas (1996). 
define the social momentary utility function: ${ }^{8}$

$$
U_{t}^{\eta}(x)=\max _{x^{1}, \ldots, x^{I}}\left\{\sum_{i=1}^{I} \eta_{i}^{-1} \delta_{i}^{t} u^{i}\left(x^{i}\right): \sum_{i=1}^{I} x^{i}=x, x^{i} \in X\right\}
$$

It is now easily verified that the aggregate competitive equilibrium allocation, $x_{t}=\sum_{i} x_{t}^{i}$, all $t$, coincides with the solution to the planning problem:

$$
\begin{aligned}
& \max \sum_{t=0}^{\infty} U_{t}^{\eta}\left(x_{t}\right) \\
& \text { s.t. }\left\{\begin{array}{l}
x_{t} \leq z_{t}-k_{t} \\
\left(-k_{t-1}, z_{t}\right) \in Y, \text { all t } \\
z_{0}>0 \text { given }
\end{array}\right.
\end{aligned}
$$

This is, of course, an optimal growth problem. Equivalently, it is the extensive form of a dynamic programming problem. However, there are two fundamental differences between (5) and homogenous programming problems as they arise in representative agent models of unbounded growth (e.g. Alvarez and Stokey (1998)). Firstly, even though $u^{i}$ is homogenous of degree $\alpha_{i}$ for all $i, U_{t}^{\eta}$ will not be homogenous unless $\alpha_{i}=\alpha$, all $i$. Secondly, $U_{t}^{\eta}$ will not be stationary - i.e., it will not be the case that $U_{t}^{\eta}(x)=\delta^{t} u^{\eta}(x)$ - unless $\delta_{i}=\delta$ for all $i$. Finally, (5) depends, of course, on $\eta$ and therefore only tracks competitive equilibria to the extend that the marginal utilities of income are known. ${ }^{9}$

Existence and Unbounded Growth

To prove existence of equilibrium a definition and a last assumption are needed:

Definition 2 The maximum factor of expansion, $\lambda_{*} \in \mathbf{R}_{+}$, is defined as the largest value for which there exists $(-k, z) \in Y$ with $z-\lambda_{*} k \geq 0$ and $z \gg 0$.

Assumption $4 \delta_{i} \lambda_{*}^{\alpha_{i}}<1$, all $i \in \mathcal{I}$, and $\|z\| \leq \lambda_{*}\|k\|$ for all $(-k, z) \in Y$, where $\|\cdot\|$ may be any norm on $\mathbf{R}^{N}$.

$\overline{8 \text { If } W^{i}}=0$, some $i$, then take $\eta^{-1}=0$ (the social planner excludes zero income consumers).

9 It should be noted that even if $\alpha_{i}=\alpha$ and $\delta_{i}=\delta$ for all $i$, instant utility function can differ and so heterogeneous consumers is not always in conflict with the homogenous programming formulation (Alvarez and Stokey (1998), Jensen (2003)). This is returned to in section 4 . 
Under assumption $1, \lambda_{*}$ will be well-defined. $\lambda_{*}$ is the maximum growth factor which $Y$ can support along a ray, since in at least one coordinate: $z_{t}^{n}=$ $\lambda_{*} k_{t-1}^{n} \leq \lambda_{*}^{t} k_{0}^{n}$, where the inequality follows recursively from market balance: $k_{t}^{n} \leq z_{t}^{n}$. Roughly put, assumption 4 places sufficient structure on (5) to make it possible to conclude by the Weierstrass' theorem that a solution exists for an arbitrary choice of marginal utilities $\eta$. There are various ways to obtain this result. One way is to use a variant of the argument in Dolmas (1996) who considers the product topology in which compactness follows from Tychonoff's theorem. Another way is to observe that assumption 4 places the set of feasible sequences in $S_{\lambda_{*}}=\left\{z=\left(z_{t}\right)_{t=0}^{\infty}: \sup _{t} \lambda_{*}^{-t}\left\|z_{t}\right\|<\infty\right\} .{ }^{10} S_{\lambda_{*}}$ therefore provides a natural choice of commodity space. In either case continuity will follow from the fact that the objective in (5) is bounded from above and below on the feasibility set. As for the latter, this follows immediately from lemma 1 below. Boundedness from above arrives from the following: If $\alpha_{i}>0$ then $\sum_{t=0}^{\infty} \delta_{i}^{t} u^{i}\left(x_{t}^{i}\right)=\sum_{t=0}^{\infty} \delta^{t}\left\|x_{t}^{i}\right\|^{\alpha_{i}} u^{i}\left(\frac{x_{t}^{i}}{\left\|x_{t}^{i}\right\|}\right)$, and since $\left\|x_{t}^{i}\right\| \leq \lambda_{*}^{t} C, C>0$, and $\delta_{i} \lambda_{*}^{\alpha_{i}}<1$, it follows that $\sum_{t=0}^{\infty} \delta_{i}^{t} u^{i}\left(x_{t}^{i}\right) \leq \sum_{t=0}^{\infty}\left(\delta_{i} \lambda_{*}^{\alpha_{i}}\right)^{t} u^{i}\left(\frac{x_{t}^{i}}{\left\|x_{t}^{2}\right\|}\right) \leq \bar{u}<+\infty$. If $\alpha_{i}<0$ then $U^{i}\left(\mathbf{x}^{i}\right)=\sum_{t=0}^{\infty} \delta_{i}^{t} u^{i}\left(x_{t}^{i}\right)=\sum_{t=0}^{\infty} \delta_{i}^{t}\left\|x_{t}^{i}\right\|^{\alpha_{i}} u^{i}\left(\frac{1}{\left\|x_{t}^{i}\right\|} x_{t}\right)<0$, since otherwise monotonicity would be violated ( $u^{i}$ is homogenous). Finally, if $\alpha_{i}=0$ one can transform $U^{i}\left(\mathbf{x}^{i}\right)$ to a homogenous of degree 1 function and modify the first argument to reach the same conclusion.

Omitting the details on how to show compactness of the feasibility set, let us simply take existence of a solution to (5) for arbitrary $\eta$ as given. ${ }^{11}$ As an expression of the second theorem of welfare economics, the solution to (5) given $\left(\eta_{1}^{-1}, \ldots, \eta_{I}^{-1}\right)$ will correspond to a competitive equilibrium for $\mathcal{E}\left(z_{0}\right)$ when the distribution of income $\left(\theta^{1}, \ldots, \theta^{I}\right)$ is chosen such that $W^{i}=\theta^{i} p_{0} z_{0}$ where $p_{t}=$ $D U_{t}\left(x_{t}\right), t=0,1,2, \ldots$ and $W^{i}=\sum_{t=0}^{\infty} p_{t} x_{t}^{i}{ }^{12}$ Now let $q^{i}\left(\eta^{-1}\right)=\sum_{t=0}^{\infty} p_{t} x_{t}^{i}-$ $\theta^{i} p_{0} z_{0}$ (where $p_{t}=D U_{t}\left(x_{t}\right)$ as before). ${ }^{13} q^{i}\left(\eta^{-1}\right)$ will be continuous, positively homogenous of degree 1 and $\sum_{i} q^{i}\left(\eta^{-1}\right)=0$, and by use of assumption 2 it is easy to show that $\frac{q^{i}\left(\eta^{-1}\right)}{\eta_{i}}, i \in \mathcal{I}$ have all properties of standard excess demand functions from finite dimensional economies. By a fixed point theorem on inwards pointing vector fields (Border (1985), chapter. 18), then follows the existence of a list of welfare weights such that $q^{i}\left(\eta^{\prime}\right)=0$ all $i$. By construction, this list of welfare weights will lead to a Pareto optimum where all consumers' budget equations are satisfied, hence to a competitive equilibrium:

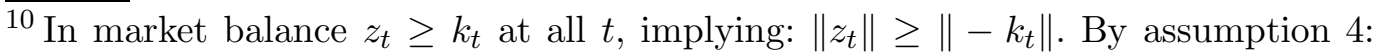
$\left\|z_{t+1}\right\| \leq \lambda_{*}\left\|-k_{t}\right\|$, so by the previous inequality, $\left\|z_{t+1}\right\| \leq \lambda_{*}\left\|z_{t}\right\|$, and hence, $\left\|z_{t+1}\right\| \leq \lambda_{*}^{t}\left\|z_{0}\right\|$; but then by market balance $\left\|x_{t}^{i}\right\| \leq\left\|z_{t}\right\| \leq \lambda_{*}^{t-1}\left\|z_{0}\right\|$.

${ }^{11}$ A detailed proof can be found in the working paper version of this paper.

${ }^{12} \mathbf{x}^{i}, i=1, \ldots, I$, can be recovered from $\mathbf{x}$ through (4) since $u^{i}, i=1, \ldots, I$, are strictly concave.

${ }^{13}$ Again, if $\eta_{i}^{-1}=0\left(\theta^{i}=0\right)$ this (dead) consumer is excluded.
} 
Theorem 1 Let the previous assumptions be satisfied. Then there exists a competitive equilibrium for all $\mathcal{E}\left(z_{0}\right), z_{0} \gg 0$.

The existence proof is a generalization of various results for the (representative agent) optimal unbounded growth model (e.g. Dolmas (1996), Alvarez and Stokey (1998)). The method of proof is natural in light of (5): To establish existence for every possible list of welfare weights and then show that some list of welfare weights corresponds to a competitive equilibrium (cf. Negishi (1960)). The next result provides a further characterization of consumers' demand in competitive equilibrium.

Lemma 1 For every consumer, $i \in \mathcal{I}$, with positive income, $W^{i}>0$, the consumption sequence satisfies the following "lower growth bound" in a competitive equilibrium:

$$
\left\|x_{t}^{i}\right\|>A\left[\left(\delta_{i} \lambda\right)^{\frac{1}{1-\alpha_{i}}}\right]^{t}, t \geq T
$$

where $A>0$ and $\lambda<\lambda_{*}$ are chosen freely, but $T \in \mathbf{N}$ (generally) depends upon their value.

Corollary 1 Assume that $\delta_{i}>\lambda_{*}^{-1}, i \in \mathcal{I}$. Then $\left\|x_{t}^{i}\right\| \rightarrow \infty$ as $t \rightarrow \infty$.

Proof: Given a production sequence $\mathbf{y}$ the firm's profit will be $\sum_{t=0}^{\infty} p_{t}\left(z_{t}-k_{t}\right)$. In equilibrium this is equal to: $\eta_{i}^{-1} p_{0} \tilde{z}_{0}+\eta_{i}^{-1} \sum_{t=0}^{\infty} \delta_{i}^{t} D u\left(x_{t}\right)\left(\bar{z}_{t}-\bar{k}_{t}\right)$, where an upper bar selects the $N^{c}$ coordinates of the consumed goods, a tilde selects the non-consumed goods, and $\eta_{i}$ is the marginal utility of income of consumer $i$. By definition, there exists $(-k, z)$ such that $-k+\lambda_{*}^{-1} z \geq 0$ with $z \gg 0$. Since $Y$ is a cone, the firm can thus choose any $\lambda<\lambda_{*}$ and: $-k_{t}=-\lambda^{t} k$, $z_{t}=\lambda^{t} z$, in $Y$, such that $\lambda^{-1} z_{t}-k_{t} \gg 0$ for all $t$. A lower bound on profits is thus: $\eta_{i}^{-1} \sum_{t=0}^{\infty} \delta_{i}^{t} D u^{i}\left(\frac{x_{t}^{i}}{\left\|x_{t}^{i}\right\|}\right)\left\|x_{t}^{i}\right\|^{\alpha_{i}-1} \lambda^{t}\left(-\bar{k}+\lambda^{-1} \bar{z}\right)$, where $\lambda^{-1} \bar{z}-\bar{k} \gg 0$. But by assumption 1, $p_{t}$ with $\left\|p_{t}\right\|=1$ is uniformly coordinatewise bounded from below in equilibrium, and it follows that for some $B>0$, profit is bounded from below by: $B \sum_{t=0}^{\infty} \delta_{i}^{t}\left\|x_{t}^{i}\right\|^{\alpha_{i}-1} \lambda^{t}$. But firms cannot earn infinite profits in equilibrium, so a contradiction will obtain unless: $\lim _{t \rightarrow \infty} \delta_{i}^{t}\left\|x_{t}^{i}\right\|^{\alpha_{i}-1} \lambda^{t}=0$, all $\lambda<\lambda_{*}$. This implies that for $\lambda<\lambda_{*}$ given and arbitrary $A>0$, there exists $T \in \mathbf{N}$ such that when $t \geq T:\left\|x_{t}^{i}\right\|>A\left[\left(\delta_{i} \lambda\right)^{\frac{1}{1-\alpha_{i}}}\right]^{t}$. The corollary follows directly from the lemma since $\delta_{i}>\left(\lambda_{*}\right)^{-1} \Leftrightarrow\left(\delta_{i} \lambda_{*}\right)^{\frac{1}{1-\alpha_{i}}}>1$.

The corollary says that if a consumer's discount factor is above the inverse of the maximum factor of expansion, then consumption will grow without bounds over time. It is a generalization of various isolated results in the literature (e.g. Jones and Manuelli (1990), Rebelo (1991), Dolmas (1996), and Kaganovich (1998)). The lemma is a sharpening of this observation. It describes the minimum average speed of expansion as being a power process and 
relates this to each consumer's preferences. Since the maximum speed of expansion is also a power process, $\lambda_{*}^{t}$, this draws a broad picture of equilibrium allocations as swinging within a band of relative growth rates.

As mentioned, the existence proof extends the framework of homogenous programs / unbounded optimal growth (e.g. Alvarez and Stokey (1998), Jensen (2003)) to a framework with heterogenous agents. Since to the best of knowledge, the heterogenous agent model of this paper has not been studied before in the literature; the previous results are therefore all new from that perspective. From a technical perspective the methods used so far are entirely standard, however.

\section{Heterogeneity and Balanced Growth}

Whenever a price sequence takes the form, $p_{t}=\frac{1}{(1+r)^{t}} p_{0}, p_{0} \gg 0$, it is said to be balanced with interest rate $r>0$. For a balanced price sequence the rate of savings of consumer $i$ is given by: ${ }^{14}$

$$
s_{i}(r)=\left(\delta_{i}(1+r)^{\alpha_{i}}\right)^{\frac{1}{1-\alpha_{i}}}
$$

From the Euler conditions (3) and strict concavity of $u^{i}$ follows that if consumer $i$ faces a balanced price sequence and $W^{i}>0$, then the consumer's demand $\mathbf{x}^{i}=\left(x_{0}^{i}, x_{1}^{i}, \ldots\right)$ will also be balanced:

$$
x_{t}^{i}=\left(1+g_{i}\right)^{t} x_{0}^{i}
$$

As may be checked, $g_{i}$ will in turn be determined from the savings rate by:

$$
1+g_{i}=s_{i}(r)(1+r)
$$

Finally, relative demand is determined from (recall that $u^{i}$ and hence $D u^{i}$ are homogenous):

$$
D u^{i}\left(x_{0}^{i}\right)=\eta_{i} p_{0}
$$

${ }^{14}$ The savings rate at date $T$ is: $s_{T}^{i}=\frac{\sum_{t=T}^{\infty} p_{t}^{c} x_{t}^{i}-p_{T}^{c} x_{T}}{\sum_{t=T}^{\infty} p_{t}^{c} x_{t}^{i}}$. Let $f^{i}$ denote the inverse of $D u^{i}$ which exists by the implicit function theorem. From (3) then: $x_{t}^{i}=\delta_{i}^{\frac{t}{1-\alpha_{i}}} \eta_{i}^{\frac{1}{\alpha_{i}-1}} f^{i}\left(p_{t}\right)$, since $f^{i}$ is homogenous of degree $\frac{1}{\alpha_{i}-1}$. Insert to get: $s_{T}^{i}=\frac{\sum_{t=T}^{\infty} \delta^{\frac{t}{1-\alpha_{i}}} p_{t} f^{i}\left(p_{t}\right)-\delta^{\frac{T}{1-\alpha_{i}}} p_{T} f^{i}\left(p_{T}\right)}{\sum_{t=T}^{\infty} \delta^{\frac{t}{1-\alpha_{i}}} p_{t} f^{i}\left(p_{t}\right)}$. Finally, insert $p_{t}=\frac{1}{(1+r)^{t}} p_{0}$, and reduce to $(7)$. 
For a given rate of interest, $r>0$, the most patient consumer group is defined via (7) as the set:

$$
\mathcal{P}(r)=\left\{i \in \mathcal{I}: s_{i}(r) \geq s_{j}(r), \text { all } j \in \mathcal{I}\right\}
$$

The correspondence $\mathcal{P}: \mathbf{R}_{++} \rightarrow 2^{\{1, \ldots, I\}}$ maps the interest rate into the subset of consumers who share the highest rate of savings. $s_{i}(r)$ will be a decreasing, constant, or increasing function depending on whether $\alpha_{i}<0, \alpha_{i}=0$, or $\alpha_{i}>0$, respectively. As a trivial consequence, two consumer's savings functions, $s_{i}(r)$ and $s_{j}(r)$, may intersect, and so it will typically be the case that $\mathcal{P}(r) \neq \mathcal{P}\left(r^{\prime}\right)$ when $r \neq r^{\prime}$. Note also that it is perfectly possible for a consumer to have a higher rate of savings than another consumer, even though his discount factor is lower. This observation is the topic of Boyd (2000) who shows how changing the production set $Y$ may lead to different consumers ending up holding almost all wealth. ${ }^{15}$ In this paper we shall consider the production set as a given throughout (just as in the usual Arrow-Debreu model).

In the following it is convenient to make explicit mentioning of the income distribution $\theta=\left(\theta^{1}, \ldots, \theta^{I}\right) \in \mathbf{S}_{+}^{I-1}$ by denoting an economy $\mathcal{E}\left(z_{0}, \theta\right)$. $\theta$ is best viewed as the initial distribution of income while at any given date $T>0$, the distribution of income will depend on past consumption. In the next definition this distinction becomes explicit since it features what we call the long-run distribution of income associated with a balanced growth equilibrium. This is further explained after the definition.

Definition $3\left(\left(\tilde{x}^{i}\right)_{i \in \mathcal{I}},(-\tilde{k}, \tilde{z}), \tilde{p}, g, r\right)$ is a balanced growth equilibrium with long-run distribution of income $\tilde{\theta} \in \mathbf{S}_{+}^{I-1}$ if: $g>0$, and $x_{t}^{i}=(1+g)^{t} \tilde{x}^{i}, i \in \mathcal{I}$, $k_{t-1}=(1+g)^{t} \tilde{k}, z_{t}=(1+g)^{t} \tilde{z}, p_{t}=\frac{1}{(1+r)^{t}} \tilde{p}, t=0,1,2, \ldots$ is a competitive equilibrium for $\mathcal{E}(\tilde{z}, \tilde{\theta})$. The allocation $\left(\left(\tilde{x}^{i}\right)_{i \in \mathcal{I}},(-\tilde{k}, \tilde{z}), g\right)$ associated with a balanced growth equilibrium is called a balanced growth path.

A thing to notice is that a balanced growth equilibrium (BGE) is a limit concept: If one considers an economy $\mathcal{E}\left(z_{0}, \theta\right)$ whose competitive equilibrium converges to a BGE, then this BGE will be a competitive equilibrium for an economy $\mathcal{E}(\tilde{z}, \tilde{\theta})$ where, in general, $\tilde{z} \neq z_{0}$ and $\tilde{\theta} \neq \theta$ (of course if $\mathcal{E}\left(z_{0}, \theta\right)$ begins exactly in the steady state, then $\theta=\tilde{\theta}$ and $z_{0}=\tilde{z}$ ). While $\theta$ corresponds to the initial distribution of income, $\tilde{\theta}$ can consequently be interpreted as the long-run distribution of income (associated with the BGE). To be precise, $\tilde{\theta}$ will approximately be equal to the distribution of income which one measure

\footnotetext{
$\overline{15}$ With unbounded growth this statement is a relative one: If all consumers' discount factors are above the inverse of the maximum factor of expansion then they will all chose consumption plans which grow without bounds (lemma 1). Still, those with the highest rate of savings will consistently save more, hence their share of the total wealth stock will approach unity as time goes to infinity.
} 
at date $t=T$ where $T$ is sufficiently large for the approximation to be a good one.

It follows immediately from (9) that $g=g_{i}$ for all $i \in \mathcal{P}(r)$. While $s_{i}(r)$ may be increasing, decreasing, or constant (see above), direct investigation of reveals that $s_{i}(r)(1+r)$ unambigously will be an increasing function of $r$. From (9) therefore follows that $g_{j}<g$ for all $j \notin \mathcal{P}(r)$ (where $g$ is the common growth rate of the most patient consumers). By comparison with definition 3 this implies the following:

Lemma 2 If $\theta^{i}>0$ for all $i$, and a competitive equilibrium for $\mathcal{E}\left(z_{0}, \theta\right)$, converges to a balanced growth equilibrium, $\left(\left(\tilde{x}^{i}\right)_{i \in \mathcal{I}},(-\tilde{k}, \tilde{z}), \tilde{p}, g, r\right)$, with long-run distribution of income $\tilde{\theta}$; then it must be the case that $\tilde{\theta}^{i}=0$ for all $i \notin \mathcal{P}(r)$.

Lemma 2 is the unbounded growth model's closest parallel to the Ramsey conjecture of the bounded growth model. Roughly it says that only the most patient consumers can be active in a BGE. Yet, while in a stationary state, the set of most patient consumers are always those with the highest discount factor, this set is endogenously determined here as will become clear from the next result. ${ }^{16}$ This result would appear to be new in the literature, and it is clearly a quite practical result both from a technical and an applied perspective because it gives a complete characterization of the set of balanced growth equilibria.

Theorem $2\left(\left(\tilde{x}^{i}\right)_{i \in \mathcal{I}},(-\tilde{k}, \tilde{z}), \tilde{p}, g, r\right)$ is a balanced growth equilibrium with distribution of income $\left(\tilde{\theta}^{1}, \ldots, \tilde{\theta}^{I}\right), \tilde{\theta}^{i} \geq 0, \sum_{i} \tilde{\theta}^{i}=1$, if and only if it satisfies the following equations:

(1) $1+g=s(r)(1+r)$

(2) $\tilde{x}^{i}=\arg \max \left\{u^{i}(x): \tilde{p} x \leq\left(1-s_{i}(r)\right) \tilde{\theta}^{i} \tilde{p} \tilde{z}\right.$ and $\left.x \in X\right\}$, when $i \in \mathcal{P}(r)$, and $\tilde{\theta}^{i}=0$, hence $x_{0}^{i}=0$, if $i \notin \mathcal{P}(r)$.

$(3)(-\tilde{k}, \tilde{z}) \in \arg \max \{\tilde{p} z-(1+r) \tilde{p} k:(-k, z) \in Y\}$.

(4) $\sum_{i=1}^{I} \tilde{x}^{i}=\tilde{z}-(1+g) \tilde{k}$

Proof: In the appendix

Theorem 3 There exists a balanced growth equilibrium.

The proof of theorem 3 is omitted, but the strategy is obvious in light of theorem 2: To show existence of a solution to the system (1)-(4). As far as the

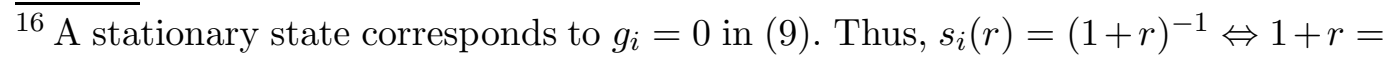
$\delta_{i}^{-1}$. It follows that $1+r=\min _{i} \delta_{i}^{-1}$ in a stationary state, since otherwise some consumers would choose $g_{i}>0$. 
latter is concerned, the next lemma (which will also be used in the example below) takes us most of the way:

Lemma 3 The system (1)-(4) satisfies "Walras' law": $\tilde{p}\left(\sum_{i=1}^{I} \tilde{x}^{i}-\tilde{z}-(1+\right.$ g) $\tilde{k})=0$.

Proof: By monotonicity of $u^{i}$ each consumer will satisfy the "budget constraint", $\tilde{p} \tilde{x}^{i}=\left(1-s_{i}(r)\right) \theta^{i} \tilde{p} \tilde{z}$, use that $\theta^{i}=0$ if $s_{i}(r) \neq s(r)$ and sum to get: $\tilde{p} \sum_{i=1}^{I} \tilde{x}^{i}=(1-s(r)) \tilde{p} \tilde{z}$. Walras' law can thus be written: $\tilde{p}[(1-s(r)) \tilde{z}+\tilde{z}-$ $(1+g) \tilde{k}]=0$. But by $(1), \frac{1+g}{s(r)}=1+r$ so this becomes: $\tilde{p} \tilde{z}-(1+r) \tilde{p} \tilde{k}$ which is the firms' profit. Since $Y$ is a cone this equals zero.

As is well known, Walras' law is helpful in showing existence of a solution to the excess demand system (4). But equally important, it says that (1)-(4) can be seen as a fictitious economy whose equilibria coincide with the set of BGEs. The bounded growth model with heterogenous consumers has a parallel "fictitious economy", which is used in that setting to show existence of a stationary steady state (cf. Bewley (1982)). Besides from the obvious difference that the interest and growth rates are endogenous in the present setting and semi-exogenous in the bounded setting $\left(g=0, r=\delta^{-1}\right.$, where $\delta$ is the highest discount factor among the consumers); the central difference is that the long-run distribution of income $\left(\tilde{\theta}^{1}, \ldots, \tilde{\theta}^{I}\right)$ is endogenously determined in a dynamically BGE since it directly enters into the fictitious economy of theorem 2. As explained at length in the introduction, this difference is of consequence. And as the following example shows, there is in general no way to isolate a "most patient" group of consumers and derive sound long-run conclusions by studying this group in isolation.

Example 1 Consider an economy with two capital goods and one consumption good: $N^{c}=1, N=2$. The production side consists of two types of firms, each of which has access to a Leontief-type activity vector. Type A firms can choose plans, $\left(-k_{t-1}^{o},-k_{t-1}^{h}, y_{t}^{o}, y_{t}^{h}\right) \in Y^{A}$, where (normalizing $\left.k^{h}=1\right)$ :

$$
Y^{A}=\left\{\left(k^{1}, k^{2}, y^{1}, y^{2}\right)=\lambda(0.85,1,1.5,1.04), \lambda \geq 0\right\}
$$

Type $B$ firms can produce according to:

$$
Y^{B}=\left\{\left(k^{1}, k^{2}, y^{1}, y^{2}\right)=\lambda(0.55,1,1.05,1.07), \lambda \geq 0\right\}
$$

There is free entry, so the production set, $Y$, is the convex cone:

$$
Y=\left\{\left(k^{1}, k^{2}, y^{1}, y^{2}\right)=\alpha y^{A}+(1-\alpha) y^{B}, y^{A} \in Y^{A}, y^{B} \in Y^{B}, \alpha \in[0,1]\right\}
$$

First note that by "Walras' law" (lemma 3), once the second market clears in the sense of (4) of the fictitious economy, i.e., $(*) z_{0}^{2}=(1+g) k_{0}^{2}$; the first (consumption-investment good) market automatically clears. Take some 
level of the interest rate, $r>0$, as given and consider the "long-run profit maximization" condition ((3) of the long-run economy) together with (*). It is easily shown that if $r<0.18$ or $r>0.46$ then it is only profitable to operate firms of type $B$ when prices are such that firms of type $B$ do not earn strictly positive profits. If $0.18<r<0.46$ the opposite is true: Only firms of type $A$ will produce since if these are to earn zero profits, type $B$ firms will earn negative profits. When $r=0.18$ or $r=0.46$ there is a continuum of relative prices which support both firm types. Now note by $(*)$ that when only type $A$ firms operate, the growth factor $1+g$ must be equal to 1.04. Similarly when firms of type $B$ produce, only then $1+g=1.07$.

Taking $r>0$ as given, (3) and $(*)$ now determine $1+g$, the growth factor, uniquely.

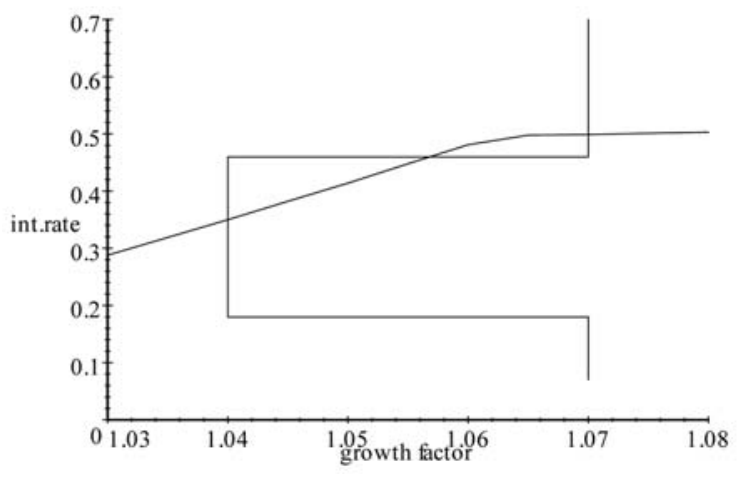

Fig. 1. Balanced Growth Diagram

The piecewise linear curve in figure 1 depicts the resulting "production side curve".

The second curve is the "consumption side curve", (1) of the fictitious economy: $1+g=s(r)(1+r)$. This derives from the following set-up: Take $I=2$, and $u^{i}(x)=\frac{1}{\alpha_{i}} x^{\alpha_{i}}, i=1,2$. Then take $\left(\delta_{1}, q_{1}\right)=(0.896,-3.86),\left(\delta_{2}, q_{2}\right)=$ $(0.677,0.76)$. By definition $s(r)=\max _{i=1,2} s_{i}(r)$, where $s_{i}(r)$ is the savings function of consumer $i$ as defined above. For each consumer we may draw a similar curve: $1+g_{i}=s_{i}(r)(1+r)$, or inserting $s_{i}(r)$ and inverting: $r=$ $\delta_{i}\left(1+g_{i}\right)^{1-\alpha_{i}}-1$. The monotone curve in the figure is the lower envelope of those two curves. For interest rates below 0.5 consumer 1 has the highest rate of savings, so "his curve lies below" that of consumer 2 and is consequently the one depicted in the figure. At $r=0.5$ the curve has a kink and flattens out to become almost horizontal. The reason is that for $r>0.5$ consumer 2 's savings rate is higher than consumer 1 's.

For every intersection between the two curves in the figure, there exists an attainable balanced growth equilibrium. ${ }^{17}$ As seen there are three such candi-

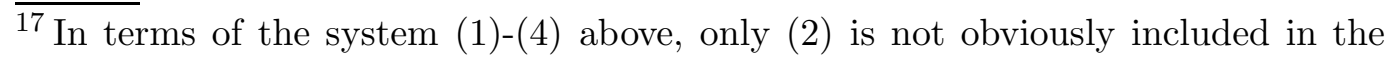


dates, but the middle one where $1+g=1.055$ can be shown to be dynamically unstable (see Jensen (2002), appendix B, the argument of which carries over to the present case). In the first stable $B G E(r=0.355$ and $g=0.04)$, consumer 1 has the highest savings rate, so $\theta^{1}=1, \theta^{2}=0$ : As the economy converges to the BGE, consumer 2 vanishes relative to consumer 1 . In the second stable $B G E(r=0.503$ and $g=0.07)$, consumer 1 vanishes and the corresponding $B G E$ has $\theta^{1}=0, \theta^{2}=1$. Note that $\delta_{1}>\delta_{2}$, so not only does the consumer with the highest factor of discount "loose" in this second stable BGE, the BGE with the highest growth rate is associated with the consumer who places lowest weight on future consumption / discounts more rapidly.

Most importantly, the example shows that varying the initial distribution of income will affect the long-run distribution of income and hence the growth and interest rate even though neither of the consumers is excluded from the economy (i.e., is given zero income).

\section{A Turnpike Theorem}

From the viewpoint of homogenous consumer models (homogenous programming), the conclusions of the previous section are discouraging. What example 1 shows is that, unlike in bounded models, one can generally not view a homogenous program as a convenient (approximate) representation of an underlying heterogenous consumer model. Still, intuition suggests that if a group of consumers becomes "sufficiently patient" relative to the rest, a homogenous program based on this group alone should be a good approximation independently of the initial distribution of income. Indeed this is so and this section's first objective is to formulate and prove a precise statement of this fact.

If for a subset $\tilde{I} \subset \mathcal{I}: \delta_{i}=\delta$ and $\alpha_{i}=\alpha<1$ all $i \in \tilde{I}, \tilde{I}$ is called a savings group. This term makes sense in the light of (7), since consumers in a savings group will have the same savings rate for all interest rates. As long as $N^{c}>1$, the consumers in such a group need not be identical, however. For example let $\# \tilde{I}=2, \delta_{1}=\delta_{2}$, and take: $u^{1}\left(x^{1}, x^{2}\right)=\left(x^{1}\right)^{\beta_{1}}\left(x^{2}\right)^{\beta_{2}}, \beta_{1}+\beta_{2}=\alpha<1$, and $u^{2}\left(x^{1}, x^{2}\right)=\left[\left(x^{1}\right)^{\gamma}+\left(x^{2}\right)^{\gamma}\right]^{\frac{\alpha}{\gamma}}$. Even if all consumers are in the same savings group, i.e., $\tilde{I}=\mathcal{I}$, heterogeneity would still affect equilibria through relative prices. In particular, varying the distribution of income will affect relative demand in a BGE, hence also the growth rate (this is clear from the fictitious economy of theorem 2 and examples to this effect are easily constructed).

curves. But since $N^{c}=1,(2)$ says simply that $p_{0}^{1} x^{1}=\left(1-s_{i}(r)\right) \theta^{i} p_{0} z_{0}$, and this equation has already been assumed to hold in the derivation of Walras' law (they imply each other given clearing of the first market and profit maximization). 
The results below concern the situation where the discount factor of some savings group is varied but the initial stock, $z_{0}$, and the initial distribution of income, $\left(\theta^{1}, \ldots, \theta^{I}\right)$, are kept fixed. To emphasize this, an economy will be denoted $\mathcal{E}^{\delta}\left(z_{0}\right)$, where $\delta$ is the discount factor of the savings group focused upon. The first result says that if the discount factor of a savings group becomes sufficiently high (the group becomes sufficiently "patient"), then the social planner problem corresponding to a competitive equilibria will eventually (as $t \rightarrow \infty)$ depend only on preferences of consumers in this group. Effectively then, the non-homogenous, non-stationary planner problem (5) will converge to a problem which is homogenously stationary.

Lemma 4 Take an arbitrary savings group $\tilde{I} \subset \mathcal{I}$ with discount factor $\delta<$ $\lambda_{*}^{-\alpha}$. Let $U_{t}^{\delta}\left(x_{t}\right)$ denote the social momentary utility function as defined in (4). There exists $\bar{\delta}<\lambda_{*}^{-\alpha}$, such that if $\delta \geq \bar{\delta}$, then for any feasible aggregate consumption sequence, $\left(x_{0}, x_{1}, x_{2}, \ldots\right):{ }^{18}$

$$
\left|U_{t}^{\delta}\left(x_{t}\right)-\delta^{t} u^{\delta}\left(x_{t}\right)\right| \rightarrow 0 \text { as } t \rightarrow \infty
$$

where $u^{\delta}$ is given by:

$$
\begin{array}{r}
u^{\delta}(x)=\max \\
\sum_{i \in \tilde{I}} \eta_{i}^{-1} u^{i}\left(x^{i}\right) \\
\text { s.t. } \sum_{i \in \tilde{I}} x^{i}=x
\end{array}
$$

and $\eta_{i}$ is the marginal utility of income of consumer $i \in \tilde{I}$.

Proof: In the appendix.

As the reader who is familiar with turnpike theory will notice, the condition of lemma 4 that a group of consumers must be "sufficiently patient" is known from the bounded growth model to imply the convergence of the equilibrium allocation to a steady state (Bewley (1982), Coles (1985), Yano (1998)). As we shall next (theorem 4), the same turns out to be true in the unbounded growth model. The results to follow are neighborhood results, i.e., they predict convergence into a suitable neighborhood rather than convergence in the asymptotic sense. With stronger assumptions on the production set $Y$, this can be strengthened to asymptotic convergence. ${ }^{19}$ Further discussion of the results follows after the statements.

$\overline{18}$ The following result may be strengthened to say that $U_{t}^{\delta}$ converges to $u^{\delta}$ in the uniform $C^{1}$ topology on feasible aggregate consumption sequences.

${ }^{19}$ See Jensen (2002), the argument of which is easily adapted to the present situation along the lines of the proof of theorem 4 . 
Theorem 4 (Dual Stability) Take an arbitrary savings group $\tilde{I} \subset \mathcal{I}$ with homogenous of degree $\alpha<1$ instant utility functions and discount factor $\delta<$ $\lambda_{*}^{-\alpha}$. Let $\left(\theta^{1}, \ldots, \theta^{I}\right) \gg 0$ be any initial distribution of income and consider the economy $\mathcal{E}^{\delta}\left(z_{0}\right)$.

For all $\alpha>0$ there exists some value $\delta_{\alpha}<\lambda_{*}^{-\alpha}$ and $T \in \mathbf{N}_{0}$ such that if $\delta \geq \delta_{\alpha}$, then, independently of $z_{0} \gg 0$, any competitive equilibrium allocation of $\mathcal{E}^{\delta}\left(z_{0}\right)$ is supported by a price sequence, $\mathbf{p}=\left(p_{t}\right)_{t=0}^{\infty}$, for which:

$$
\left\|\hat{p}_{t}-p\right\| \leq \alpha, \quad \text { all } t \geq T
$$

Where $\hat{p}_{t}=\frac{1}{(1+r)^{t}} p_{t}$, and $r$ and $p$ are the interest rate and price vector of a balanced growth equilibrium of $\mathcal{E}^{\delta}\left(z_{0}\right)$ with $p$ normalized such that $\|p\|=1$.

Proof: In the appendix.

In words, theorem 4 says that if we fix a savings group and increase this group's discount factor $\delta$ such that the group places sufficiently strong weight on future consumption; then eventually the economy's prices will be close to those of a BGE. The intuition is as follows: When $\delta$ becomes sufficiently high for some savings group, we know from lemma 4 that the Negishi-type planner's problem will approximate that of this group alone. This statement is independent of the initial distribution of income. Raising $\delta$ is the same as placing relatively stronger weight on future consumption. In an environment with unbounded growth, the growth rate is a good "proxy" for future consumption: If one places a lot of weight on the future, the relative allocation of consumption within periods becomes of secondary importance compared to the scale (which is determined by the growth rate). The deep insight of turnpike theory is the observation (first made by Dorfman, Samuelson and Solow (1958), chapter 11, see also McKenzie (1976)) that the steady states are in this connection "the single most efficient way for the system to grow" (Dorfman et al., op.cit., p.11). Hence the system will be attracted to a steady state, which here means a balanced growth path (whose supporting prices are exactly those of theorem 4). ${ }^{20}$ Mathematically, the previous observations translate into a value loss and Liapounov function, respectively. The interested reader should consult the proof for details.

Corollary 2 (Neighborhood Turnpike) For $\alpha>0$, take $\delta_{\alpha}$ and $T$ as in

\footnotetext{
$\overline{{ }^{20} \text { Note }}$ that based on this intuition we would expect that in the limit as $\delta \rightarrow \lambda_{*}^{-\alpha}$, consumers simply seek to maximize the growth rate. And this is indeed true as can be seen already from lemma 1 by choosing $\delta_{i}=\delta=\lambda^{-\alpha}$ and letting $\lambda \rightarrow \lambda_{*}$ (recall that $\lambda_{*}$ is the maximum factor of growth).
} 
theorem 4. Then, if $\delta \geq \delta_{\alpha}$ :

$$
\left\|c_{i} \hat{x}_{t}^{i}-\tilde{x}^{i}\right\| \leq \alpha
$$

for all $t \geq T$, where $c_{i}$ is a positive constant, $\hat{x}_{t}=\left(1+g_{i}\right)^{-t} x_{t}^{i}, t=0,1,2, \ldots$, $1+g_{i}=s_{i}(r)(1+r), \tilde{x}^{i}$ solves $\tilde{p}^{c}=D u^{i}\left(\tilde{x}^{i}\right), i \in \mathcal{I}$, and $r$ and $\tilde{p}^{c}$ are those of the balanced growth equilibrium of theorem 4 .

Proof: The corollary follows immediately from (7), (8), and (9), and the Euler conditions, $\eta_{i} p_{t}^{c}=D u^{i}\left(x_{t}^{i}\right), t=0,1,2, \ldots$, since $D u^{i}$ is a continuous, invertible function.

The previous results are normally referred to as "dual" theorems (Yano (1998)) since they concern prices and consumption plans, not production plans. In order to ensure that production plans also converge to the neighborhood of a BGP; stronger assumptions are necessary in order to rule out certain cyclic patters. We shall not go into the details but refer the reader to Yano (1998) and Jensen (2003).

The first turnpike theorem in an unbounded growth set-up with a Ramseytype objective is that of Kaganovich (1998). Kaganovich considers the case with a representative agents and a production set which exhibits no joint production. Jensen (2003) considers an arbitrary production set, in particular joint production is allowed. ${ }^{21}$ Theorem 4 can be seen as an integration of these representative agent results with intertemporal general equilibrium theory (Bewley (1982), Coles (1985)). Alternatively, theorem 4 can be seen as an independent result addressing non-homogenous programs by showing that, under certain circumstances, they will approach homogenous programs as time goes to infinity. As the proof shows, this is a non-trivial extension of existing results.

\section{Concluding Remarks}

Throughout the previous sections, a central theme was that with unbounded growth the long-run distribution of income is endogenous in a fundamental way: Different initial distributions of income generally lead to different balanced growth equilibria with different associated long-run distributions of income. This should be compared with the bounded growth model (Bewley

\footnotetext{
${ }^{21}$ But the cost is that the results become dual neighborhood results. To what extend asymptotic convergence can be ensured without excessive assumption on the production technology (such as $Y$ being a strictly convex cone as assumed in Jensen (2002)) remains an open question.
} 
(1982), Yano (1998)), where the consumers with the highest discount factors eventually will end up holding all wealth, and so the long-run distribution of income is exogenous for all practical purposes.

Of course, an equilibrium need not converge into the neighborhood of a balanced growth equilibrium (BGE) in the first place, a topic which the turnpike theorem of section 4 sought to address. If a group of consumers places sufficiently high weight on future consumption, then equilibrium prices will eventually be in the neighborhood of a balanced price "turnpike".

Importantly, in a BGE only preferences of those consumers who are among the most patient will influence market prices (see section 3). But this observation is the closest parallel to the Ramsey conjecture which the unbounded growth model has on offer.

Imagine an economy along the lines of this paper but with two countries each of which is described by a representative consumer. One country's agents happen to be relatively less patient than the other country's at the ruling world rate of interest. The faster growing country will then completely determine relative world market prices including prices in the relatively less developed country. What example 1 of section 3 shows is that in such a situation, a redistribution of resources may turn the situation around so that the less developed economy becomes the faster growing one and remains so indefinitely because the economy converges to a new BGE where it determines market prices.

I believe that this two-country story points to possibilities which make worth the effort of studying heterogenous consumers in the unbounded growth scenario. The mathematics used is, occasionally, somewhat involving, but it does lead us to conclusions which the representative agent set-up is completely incapable of addressing. As such, I hope that the results have contributed to an understanding of heterogeneity which makes future research on the topic appealing. In particular, it might be interesting to construct better and more intuitive examples of "unequal" growth and try to test some of the implications empirically.

\section{A Proof of theorem 2}

The proof is based on two lemmas.

Lemma A.1 A solution to the fictitious economy (1)-(4) for which $s(r)<1$ is a competitive equilibrium, hence a balanced growth equilibrium. 
Proof: As explained in section 3, given a balanced price sequence, $p_{t}=\frac{1}{(1+r)^{t}} \tilde{p}$, (8)-(10) are equivalent to the infinite sequence of Euler conditions of consumer i. By (8) and (9): (10) and the budget condition, $\sum_{t} p_{t} x_{t}^{i}=W^{i}=\theta^{i} \tilde{p} z_{0}$ are therefore necessary and sufficient for $x_{t}^{i}=\left(1+g_{i}\right)^{t} \tilde{x}^{i}$ to solve the consumer's problem where $g_{i}$ is given by (9). The problem (2) in the theorem, has an interior solution if $\theta^{i}>0$. Otherwise the solution is $\tilde{x}=0$. In the former case the first order condition,

$$
D u^{i}\left(\tilde{x}^{i}\right)=\alpha_{i} \tilde{p}
$$

must hold. But if budget balance also holds which, inserting what is known so far, is the case if and only if,

$$
\sum_{t} \frac{\left(1+g_{i}\right)^{t}}{(1+r)^{t}} \tilde{p} \tilde{x}^{i}=\theta^{i} \tilde{p} \tilde{z}
$$

then $\alpha_{i}$ must be equal to $\eta_{i}$ (the marginal utility of income). Thus if (A.2) holds, the balanced consumption sequence solves the consumer's infinite horizon maximization problem. But by (9): $\frac{g_{i}}{1+r}=s_{i}(r)$, hence if $s_{i}(r)<1$, (A.2) is equivalent to the budget condition of the problem $(2): \tilde{p} \tilde{x}^{i}=\left(1-s_{i}(r)\right) \theta^{i} \tilde{p} \tilde{z}$.

The firm will maximize profits at date $t$ if it maximizes $p_{t} z-p_{t-1} k$, over those $(-k, z) \in Y$. Inserting $p_{t}=\frac{1}{(1+r)^{t}} \tilde{p}$, and canceling out, (3) results. Given a solution, $(-\tilde{k}, \tilde{z})$, at date 0 , say, it is clear that when prices are balanced, for any $\lambda>0: \lambda^{t}(-\tilde{k}, \tilde{z}) \in Y$ will maximize profits at date $t(Y$ is a cone, and profits is zero).

Finally, since either $\theta^{i}=0$ and then $\tilde{x}^{i}=0$, or the above description applies, in particular $g=g_{i}$, all $i \in \mathcal{P}(r)$; picking the expansion factor of the firm, $\lambda$ equal to $g$, market balance at date $t$ reads: $(1+g)^{t} \sum_{i} \tilde{x}^{i}=(1+g)^{t} \tilde{z}+(1+g)^{t+1} \tilde{k}$ which is equivalent to (4) (at all $t$ ).

The previous construction explicitly verifies the individual parts of the definition of a competitive equilibrium, and since the equilibrium is balanced and attainable the proof is finished.

Lemma A.2 $s_{i}(r) \geq 1$, some $i \in \mathcal{I}$, is impossible in an attainable balanced growth equilibrium.

Proof: If $s_{i}(r) \geq 1$, some $i \in \mathcal{I}$, then $s_{i}(r) \geq 1$ for some $i \in \tilde{I}$. By (1) this implies that $1+g \geq 1+r$. By (3) then: $\tilde{p} \tilde{z}-(1+r) \tilde{p} \tilde{k}=0 \Rightarrow \tilde{p}[\tilde{z}-(1+g) \tilde{k}] \leq 0$. But by $(4): \tilde{z}-(1+g) \tilde{k} \geq 0$, and since $\tilde{p} \gg 0$ (assumption 1) then $\tilde{z}-(1+g) \tilde{k}=0$. From (4) therefore, $\sum_{i} \tilde{x}^{i}=0$ all $i$, which is a contradiction. 
Lemma A.1 says that a solution to (1)-(4) for which $\theta^{i}>0$ if and only if $i \in \mathcal{P}(r)$ is a BGE provided that the solution has $s(r)<1$ (since then $s_{i}(r)<1$ for all $\left.i\right)$. Conversely, take an arbitrary BGE. It is straight-forward to show that (1), (3), and (4) will hold (the argument in the proof of lemma A.1 consists of equivalent statements). Hence $s_{i}(r)<1$, all $i$ since the proof of lemma A.2 uses only (3) and (4) (and the trivially satisfied $\tilde{x}^{i} \geq 0$ ). Using this also (2) can be derived by the argument of lemma A.1, and theorem 2 has been proved.

\section{B Proof of lemma 4}

Consider the social maximization problem at date $t$, slightly rewritten (but equivalent):

$$
\begin{gathered}
U_{t}^{\delta}(x)=\delta^{t}\|x\|^{\alpha} \max \\
\sum_{j=1}^{I} \eta_{j}^{-1}\left(\frac{\delta_{j}}{\delta}\right)^{t}\|x\|^{\alpha_{j}-\alpha} u^{j}\left(\frac{1}{\|x\|} x^{j}\right) \\
\text { s.t. } \sum_{j=1}^{I} x^{j}=x
\end{gathered}
$$

First consider a consumer $j$ with $\alpha_{j}>\alpha, \alpha_{j}>0$. Since the maximum factor of expansion $\lambda_{*}$ bounds consumption growth in a norm $\|\cdot\|$ by assumption 4 (see the argument just after the assumption), and $u^{i}\left(\frac{1}{\|x\|} x^{j}\right)$ is bounded from above, there exists $A>0$ such that: $\eta_{j}^{-1}\left(\frac{\delta_{j}}{\delta}\right)^{t}\|x\|^{\alpha_{j}-\alpha} u^{j}\left(\frac{1}{\|x\|} x^{j}\right) \leq A \eta_{j}^{-1}\left(\frac{\delta_{j}}{\delta}\right)^{t} \lambda_{*}^{t\left(\alpha_{j}-\alpha\right)}=$ $A \eta_{j}^{-1}\left[\frac{\delta_{j} \lambda_{*}^{\alpha_{j}}}{\delta \lambda_{*}^{\alpha}}\right]^{t}$. But by assumption $4, \delta_{j} \lambda_{*}^{\alpha_{j}}<1$, hence there exists $\delta_{1}$, such that if $\delta>\delta_{1}$, then $\delta \lambda_{*}^{\alpha}<1$ while $\delta_{j} \lambda_{*}^{\alpha_{j}}<\delta \lambda_{*}^{\alpha}$. For such a $\delta$ therefore: $\left[\frac{\delta_{j} \lambda_{*}^{\alpha} j_{j}}{\delta \lambda_{*}^{\alpha}}\right]^{t} \rightarrow 0$ as $t \rightarrow \infty$. If $\alpha_{j}>\alpha$, but $\alpha_{j}<0$ (thus necessarily $\alpha<0$ ) then: $0 \geq \eta_{j}^{-1}\left(\frac{\delta_{j}}{\delta}\right)^{t}\|x\|^{\alpha_{j}-\alpha} u^{j}\left(\frac{1}{\|x\|} x^{j}\right) \geq \eta_{j}^{-1}\left(\frac{\delta_{j}}{\delta}\right)^{t}\left\|x^{j}\right\|^{\alpha_{j}-\alpha} u^{j}\left(\frac{1}{\left\|x^{j}\right\|} x^{j}\right)$. Surpassing the details (see e.g. Jensen (2002), appendix C), it may be shown that the individual feasibility sets can be restricted to such sets for which $\frac{1}{\left\|x^{j}\right\|} x^{j}$ is uniformly coordinatewise bounded from below. Note the intuition: The planner will not choose such plans because social utility would approach $-\infty$. This implies the conclusion since then $u^{j}\left(\frac{1}{\left\|x^{j}\right\|} x^{j}\right)$ is bounded from below. If $\alpha_{j}=0$, transform the objective with $\exp (\cdot)$ to get a homogenous function and use one of the previous arguments. Finally, for consumers with $\alpha_{j} \leq \alpha,\|x\|^{\alpha_{j}-\alpha}$ is clearly bounded from above provided that $\|x\|$ is bounded from below. So again $\delta$ may be picked sufficiently large such that $\left(\frac{\delta_{j}}{\delta}\right)^{t} \rightarrow 0$, as $t \rightarrow \infty$. 


\section{Proof of theorem 4}

Pick an initial stock $z_{0} \gg 0$, and an initial distribution of income $\left(\theta_{1}, \ldots, \theta_{I}\right) \gg$ 0 and keep these fixed throughout. The discount factor $\delta<\lambda_{*}^{-\alpha}$ will be varied it is convenient to denote the economy by $\mathcal{E}^{\delta}$. Define,

$$
U_{t}(x)=\max \left\{\sum_{i=1}^{I} \eta_{i}^{-1} \delta_{i}^{t} u^{i}\left(x^{i}\right): \sum_{i=1}^{I} x^{i}=x\right\}
$$

and,

$$
V_{t}(x)=\max \left\{\sum_{i \in \tilde{I}} \eta_{i}^{-1} \delta^{t} u^{i}\left(x^{i}\right): \sum_{i \in \tilde{I}} x^{i}=x\right\}
$$

It has been shown that if $\delta$ is picked sufficiently close to $\lambda_{*}^{-\alpha}$ then for all $\epsilon>0$, there exists $T \in \mathbf{N}$ such that $t \geq T$ implies that $\left|U_{t}\left(x_{t}\right)-V_{t}\left(x_{t}\right)\right|<\epsilon$, when $x_{t}$ lays in the feasibility set $X_{t}=\left\{x \in \mathbf{R}_{+}^{N_{c}}:\|x\| \leq A \lambda_{*}^{t}\right\}$, some $A>0$.

For the optimal growth problem with objective function $\sum_{t=0}^{\infty} V_{t}\left(x_{t}\right)$ and production set $Y$, there exists a balanced growth equilibrium by theorem 3 . Denote this by $(\tilde{x},-\tilde{k}, \tilde{z}, \tilde{p}, g, r)$. When we wish to stress the dependence on $\delta$ we shall say that this BGE is induced by $\delta$ ( $g$ is the growth rate induced by $\delta$, etc.). Note that $\tilde{x}$ is aggregate consumption at date 0 of the consumers in $\tilde{I}$. To simplify notation, set $\gamma_{x}=1+g$, and $\gamma_{p}=(1+r)^{-1}$. Note that the consumption and price paths then can be written simply: $\tilde{x}_{t}=\gamma_{x}^{t} \tilde{x}$ and $\tilde{p}_{t}=\gamma_{p}^{t} \tilde{p}$. Let $\tilde{\Delta}=\left\{\delta \gamma_{x}^{\alpha}: \delta<\lambda_{*}^{-\alpha}\right.$ and $\gamma_{x}$ is the rate of growth induced by $\left.\delta\right\} . \hat{\rho}=\delta \gamma_{x}^{\alpha}$ is called the implicit rate of discount associated with the balanced growth equilibrium. By lemma 2 and the definition of $s(r)$ (both are of course valid also for the situation with the consumers in $\tilde{I}): \gamma_{x}=s(r)(1+r)=(\delta(1+r))^{\frac{1}{1-\alpha}} \Leftrightarrow$ $\delta \gamma_{x}^{\alpha-1}=\gamma_{p} \Leftrightarrow \hat{\rho}=\gamma_{p} \gamma_{x}$. Define,

$$
\bar{u}_{t}(x)=\max \left\{\sum_{i=1}^{I} \eta_{i}^{-1}\left(\frac{\delta_{i}}{\delta}\right)^{t} \gamma_{x}^{-\alpha t} u^{i}\left(x^{i}\right): \sum_{i=1}^{I} x^{i}=x\right\}
$$

and,

$$
\bar{v}_{t}(x)=\max \left\{\sum_{i \in \tilde{I}} \eta_{i}^{-1} \gamma_{x}^{-\alpha t} u^{i}\left(x^{i}\right): \sum_{i \in \tilde{I}} x^{i}=x\right\}
$$

Clearly now $\left|\delta^{t} \gamma_{x}^{\alpha t} u_{t}\left(x_{t}\right)-\delta^{t} \gamma_{x}^{\alpha t} v_{t}\left(x_{t}\right)\right| \rightarrow 0$ as $t \rightarrow \infty$ if $\delta$ is sufficiently large. For a competitive equilibrium consumption plan, $x_{t}, t=0,1,2, \ldots$, define $\hat{x}_{t}=$ $\gamma_{x}^{-t} x_{t}$ and likewise for $x_{t}^{i}$ (where $\gamma_{x}$ is the growth factor determined above). Clearly then, $\bar{u}_{t}\left(x_{t}\right)=u_{t}\left(\hat{x}_{t}\right) \equiv \max \left\{\sum_{i=1}^{I} \eta_{i}^{-1}\left(\frac{\delta_{i}}{\delta}\right)^{t} \gamma_{x}^{\left(\alpha_{i}-k\right) t} u^{i}\left(\hat{x}_{t}^{i}\right): \sum_{i=1}^{I} \hat{x}_{t}^{i}=\right.$ $\left.\hat{x}_{t}\right\}$, and $\bar{v}_{t}\left(x_{t}\right)=v\left(\hat{x}_{t}\right) \equiv \max \left\{\sum_{i \in \tilde{I}} \eta_{i}^{-1} u^{i}\left(\hat{x}_{t}^{i}\right): \sum_{i \in \tilde{I}} \hat{x}_{t}^{i}=\hat{x}_{t}\right\}$. This leads to the final version of the approximation result: For $\delta$ sufficiently large and $\hat{x}_{t} \in \hat{X}_{t}=\left\{x \in \mathbf{R}_{+}^{N_{c}}:\|x\| \leq A\left(\frac{\gamma_{x}}{\lambda_{*}}\right)^{t}\right\}$, all $t$, some $A>0$ :

$$
\delta^{t} \gamma_{x}^{\alpha t}\left|u_{t}\left(\hat{x}_{t}\right)-v\left(\hat{x}_{t}\right)\right| \rightarrow 0 \text { as } t \rightarrow \infty
$$


The idea of the turnpike proof is to compare value losses between $u_{t}$ and the balanced growth equilibrium arising with objective $\sum_{t} V_{t}\left(x_{t}\right)$ described above. Clearly, such a comparison generally does not lead to positive value loss functions, since the aggregate consumption sequence of the balanced growth equilibrium is associated with a different objective function (hence different support prices) in the heterogenous consumer economy. Lemma 4 states, however, that when $\delta$ and $t$ are sufficiently large, the social planner's solution is "approximately" the same whether $V_{t}$ or $U_{t}$ is used as the momentary social utility function. Therefore, since the balanced growth equilibrium of the economy consisting only of the consumers $\tilde{I}$, is indeed superior in value to all comparison paths for that economy; in the limit, the same result carries over when comparing paths from the heterogenous consumer economy with the facet. To use $u_{t}$ and $v$ to define value losses, these need to be bounded for fixed $x$ over all $\delta \leq \lambda_{*}^{-\alpha}$, i.e., must have well-defined limits as $\delta \rightarrow \lambda_{*}^{-\alpha}$. The next lemma solves this problem for $v$. The result is non-trivial.

Lemma C.1 Given $\delta$ consider $\frac{\eta_{i}}{\eta_{j}}$ (the relative marginal utilities of income between two consumers $i, j$ in a competitive equilibrium for $\left.\mathcal{E}^{\delta}\right)$. There exists $A$ and $B$ such that $0<A \leq \frac{\eta_{i}}{\eta_{j}} \leq B<+\infty$, for all $i, j \in \tilde{I}$ and $\delta<\lambda_{*}^{-\alpha}$.

Proof: By strict concavity of $u^{i}, D^{2} u^{i}(x)$ has full rank. Consequently, an inverse function $f^{i}: \mathbf{R}_{++}^{N_{c}} \rightarrow \mathbf{R}_{+}^{N_{c}}$ such that $D u^{i}(x)=y \Leftrightarrow f^{i}(y)=x$ exists by the implicit function theorem. By Euler's law, Du ${ }^{i}(x)$ is homogenous of degree $\alpha-1<0$, hence: $f^{i}(y)=x \Leftrightarrow f^{i}\left(\lambda^{\alpha-1} y\right)=\lambda x$, i.e., $f^{i}$ is homogenous of degree $\frac{1}{\alpha-1}$. It may be checked that the latter claim is valid also when $k=0$ ( $u^{i}$ log-linear). Under the assumptions $W^{i}>0$, so the Euler conditions apply and have a solution:

$$
x_{t}^{i}=\eta_{i}^{\frac{1}{\alpha-1}}\left(\delta^{-t}\left\|p_{t}\right\|\right)^{\frac{1}{\alpha-1}} f^{i}\left(\frac{p_{t}}{\left\|p_{t}\right\|}\right), t=0,1,2, \ldots
$$

Insert in the budget constraint, $\sum_{t} p_{t} x_{t}^{i}=W^{i}$, and isolate $\eta_{i}^{\frac{1}{\alpha-1}}$ :

$$
\eta_{i}^{\frac{1}{\alpha-1}}=\frac{W^{i}}{\sum_{t=0}^{\infty}\left(\delta^{-t}\left\|p_{t}\right\|^{k}\right)^{\frac{1}{\alpha-1}} f^{i}\left(\frac{p_{t}}{\left\|p_{t}\right\|}\right) \frac{p_{t}}{\left\|p_{t}\right\|}}
$$

Take two consumers, $i, j \in \tilde{I}$, and divide the respective forms of the previous expression:

$$
\left(\frac{\eta_{i}}{\eta_{j}}\right)^{\frac{1}{\alpha-1}}=\frac{W^{i}}{W^{j}} \frac{\sum_{t=0}^{\infty}\left(\delta^{-t}\left\|p_{t}\right\|^{k}\right)^{\frac{1}{\alpha-1}} f^{j}\left(\frac{p_{t}}{\left\|p_{t}\right\|}\right) \frac{p_{t}}{\left\|p_{t}\right\|}}{\sum_{t=0}^{\infty}\left(\delta^{-t}\left\|p_{t}\right\|^{k}\right)^{\frac{1}{\alpha-1}} f^{i}\left(\frac{p_{t}}{\left\|p_{t}\right\|}\right) \frac{p_{t}}{\left\|p_{t}\right\|}}
$$


$\frac{W^{i}}{W^{j}}=\frac{\theta^{i}}{\theta^{j}}$ is obviously independent of $\delta$. Even if consumers' income were not assumed proportional, for example by letting consumer $i$ hold part of the initial stock $z_{0}^{i} \gg 0, \sum_{i} z_{0}^{i}=z_{0}$, this term would be uniformly bounded from above and below since $p_{0}$ can be normalized to unit length. By assumption 1, $\tilde{P}(Y)$ is a closed subset of $S_{++}^{2 N-1}$, hence $\frac{p_{t}}{\left\|p_{t}\right\|}$ is uniformly coordinatewise bounded from above and below. Therefore there exist, $0<A<B<+\infty$ such that $A<f^{i}\left(\frac{p_{t}}{\left\|p_{t}\right\|}\right) \frac{p_{t}}{\left\|p_{t}\right\|}<B$ and $A<f^{j}\left(\frac{p_{t}}{\left\|p_{t}\right\|}\right) \frac{p_{t}}{\left\|p_{t}\right\|}<B$. Comparing with (C.4) this implies the conclusion, since the summations can be canceled out after evaluating upwards and downwards with $A$ and $B$.

Similarly to $\hat{x}_{t}$, define the "detrended" competitive equilibrium price sequence, $\hat{p}_{t}=\gamma_{p}^{-t} p_{t}$. Then for $a>0$ let:

$$
L_{t}^{a}=-\left(a^{r-1} \hat{p}_{t}-\tilde{p}\right)\left(a \hat{k}_{t}-\tilde{k}\right)
$$

and from this the Liapounov function

$$
L_{t}=\min _{\alpha \geq 0} L_{t}^{\alpha\left\|\hat{p}_{t}\right\|^{\frac{1}{1-r}}}
$$

$L_{t}^{a}$ is of course well defined for all $t$ and $\hat{\rho}$. In a moment it will be shown that there exists $T \in \mathbf{N}_{0}$ such that $t \geq T$ implies $L_{t}^{a} \geq 0$, all $a>0$. Since therefore $L_{t}^{a} \rightarrow+\infty$ as $a \rightarrow 0$ or $a \rightarrow+\infty$ (compare with (C.5)), the minimizer of (C.6) can be sought within a compact interval and $L_{t}$ is then well-defined by Weierstrass' theorem. Finally, let $\alpha_{t}$ be given implicitly by: $L_{t}=L_{t}^{\alpha_{t}\left\|\hat{p}_{t}\right\|^{\frac{1}{1-r}}}$ (the $\alpha$ that is a minimizer in (C.6)).

The value loss in (detrended) BGE prices is:

$$
G_{t}^{a}=\left(u_{t}\left(a \hat{x}_{t}\right)-\tilde{p} a \hat{x}_{t}\right)-(v(\tilde{x})-\tilde{p} \tilde{x})+a\left(\tilde{p} \hat{z}_{t}-\hat{\rho}^{-1} \tilde{p} \hat{k}_{t-1}\right)
$$

and measured in (detrended) competitive equilibrium prices:

$$
\hat{G}_{t}^{a}=\left(u_{t}\left(a \hat{x}_{t}\right)-a^{r} \hat{p}_{t} \hat{x}_{t}\right)-\left(v(\tilde{x})-a^{r-1} \hat{p}_{t} \tilde{x}\right)-a^{r-1}\left(\hat{p}_{t} \tilde{z}-\hat{\rho}^{-1} \hat{p}_{t-1} \tilde{k}\right)
$$

$G_{t}^{a} \leq 0$ and $\hat{G}_{t}^{a} \geq 0$, all $a>0$ if $t$ is sufficiently large. Indeed, multiply throughout the value loss functions with $\delta^{t} \gamma_{x}^{\alpha t}$ and note that by (C.1), for $t \rightarrow \infty$, the value loss converges to $\delta^{t} \gamma_{x}^{\alpha t}$ times the value loss function with $u_{t}$ replaced by $v$. But if $u_{t}$ is replaced by $v$ in (C.7) and (C.8), the postulated sign are simply expressing the fact that prices are supporting the detrended allocation in the sense of a separating hyperplane theorem. For details the reader is referred to Jensen (2002), appendix C, lemma 7. In conclusion, there exists $T \in \mathbf{N}_{0}$ such that $G_{t}^{a} \leq 0$ and $\hat{G}_{t}^{a} \geq 0$, for all $a>0$ and $t \geq T$. The next 
lemma is somewhat difficult to prove, because in the unbounded growth model it cannot be used at this point that $L_{t}$ is bounded (it is not necessarily welldefined without the following result). Fortunately, the proof in Jensen (2003), goes through without any substantial modifications because when considering $\hat{G}_{t+1}^{a}-G_{t+1}^{a}$, the "problematic term" $u_{t+1}\left(a \hat{x}_{t+1}\right)$ cancels out.

\section{Lemma C.2}

$$
\hat{\rho}^{-1} L_{t}^{a}-L_{t+1}^{a}=\hat{G}_{t+1}^{a}-G_{t+1}^{a}
$$

for all $a>0, t \in \mathbf{N}_{0}$, and $\delta \in \Delta$. Moreover, there exists $T \in \mathbf{N}_{0}$ such that:

$$
L_{t}^{a} \geq 0
$$

for all $a>0$ and $t \geq T$, in particular, $L_{t} \geq 0$, when $t \geq T$.

The next important step in the proof is to show that $L_{t}$ is uniformly bounded from above (by the previous lemma, it will eventually be uniformly bounded from below by 0$)$.

Lemma C.3 There exists (a uniform upper bound) $\bar{L}>0$ such that $L_{t} \leq \bar{L}$ for $\delta<\lambda_{*}^{-\alpha}$ and all $t$ larger than some $T \in \mathbf{N}_{0}$.

Proof: Since $L_{t} \leq L_{t}^{a}$, for all $a \geq 0$ (it is the minimum):

$$
L_{t} \leq-\left(\alpha^{r-1} \frac{\hat{p}_{t}}{\left\|\hat{p}_{t}\right\|}-\tilde{p}\right)\left(\alpha\left(\frac{1}{\left\|\hat{p}_{t}\right\|}\right)^{\frac{1}{r-1}} \hat{k}_{t}-\tilde{k}\right)
$$

for all $\alpha \geq 0$. Normalize $\|\tilde{p}\|=1$ and $\|\tilde{k}\|=1$. By assumption $1, \frac{\hat{p}_{t}}{\left\|\hat{p}_{t}\right\|}=\frac{p_{t}}{\left\|p_{t}\right\|}$ is uniformly bounded from below, hence by the previous normalization there exists a fixed $\alpha$ such that: $\alpha^{r-1} \frac{\hat{p}_{t}}{\left\|\hat{p}_{t}\right\|}-\tilde{p} \geq 0$, all $t, \delta$. Since by the previous lemma there exists $T \in \mathbf{N}$ such that $L_{t}^{a} \geq 0$, for all $a$ and $t \geq T$ :

$$
L_{t}^{\bar{\alpha} a_{t}} \leq\left(\alpha_{t}^{r-1} \frac{\hat{p}_{t}}{\left\|\hat{p}_{t}\right\|}-\tilde{p}\right) \tilde{k}
$$

Under the previous normalization of $\tilde{k}$ this implies the conclusion of the lemma, since all entries are then uniformly bounded.

From this point on, the proof of the theorem runs exactly as in Jensen (2003). Of course in each of the lemmas along the way the addition "for $t \geq T$ ", where $T \in \mathbf{N}$ is needed. What is happening is intuitively the following: The unbounded optimal growth problem with $\sum_{t} V_{t}\left(x_{t}\right)$ as objective has the turnpike property if $\delta \geq \delta_{\alpha}$, and $\alpha$ is the price neighborhood in the sense of (14). 
Moreover, this property (convergence into the neighborhood of a balanced price sequence) hold independently of the initial stock $z_{0} \gg 0$ and the initial distribution of income $\theta \gg 0$. When considering the heterogenous consumer case with $\delta$ sufficiently high in the sense of lemma 4 ; the value loss computed from some date $T$ and onwards converges to the value loss of the optimal growth problem with objective $\sum_{t=T}^{\infty} V_{t}\left(x_{t}\right), Y$ as production set, and $z_{T}$ as initial stock, where $z_{T}$ is the competitive equilibrium stock at date $T$. But then, by the turnpike property's independence of $z_{T}$, the value losses must be equal in the limit $T=+\infty$. This is why the value loss lemma of Jensen (2003) goes through; and once this is proved, it is straight forward to show that the competitive equilibrium price sequence must visit the balanced price sequence's neighborhood at least once (visit lemma). At this point lemma 5.2 comes into play, because when the price vector is close to the balanced price sequence, the Liapounov function is close to 0 ; and so also the value loss is close to 0 by lemma 5.2 (this may sound obvious, but it is tricky to show it in the unbounded growth set-up). Finally, this leads to the "trapping" of the price sequence in the neighborhood, hence to the statement of the theorem.

\section{References}

Alvarez, F. and N.L. Stokey, 1998, Dynamic Programming with Homogenous Functions, Journal of Economic Theory 82, 167-189.

Becker, R., 1980, On the long-Run Steady State in a Simple Dynamic Model of Equilibrium with Heterogenous Households, Quarterly Journal of Economics 95, 375-382.

Bewley, T.F., 1982, An Integration of Equilibrium Theory and Turnpike Theory, Journal of Mathematical Economics 10, 233-267.

Border, K.C., 1985, Fixed Point Theorems with Applications to Economics and Game Theory (Cambridge University Press, New York).

Boyd, J.H., 2000, Sustained Growth with Heterogeneous Households, Mimeo.

Coles, J.L., 1985, Equilibrium Turnpike Theory with Constant Returns to Scale and Possibly Heterogenous Discount Factors, International Economic Review 26, 671-679.

Dolmas, J., 1996, Endogenous Growth in Multisector Ramsey Models, International Economic Review 37, 403-421.

Dorfman, R., P. Samuelson and R. Solow, 1958, Linear Programming and Economic Analysis (McGraw-Hill, New York).

Jensen, M.K., 2002, Balanced Growth, Dynamic Stability, and the Turnpike, Ph.D. Dissertation, University of Copenhagen.

Jensen, M.K., 2003, On the Global Stability of Unbounded Optimal Growth Models, Mimeo.

Jones, L.E. and R. Manuelli, 1990, A Convex Model of Equilibrium Growth: Theory and Policy Implications, Journal of Political Economy 98, 1008-1038. 
Kaganovich, M., 1998, Sustained Endogenous Growth with Decreasing Returns and Heterogenous Capital, Journal of Economic Dynamics and Control 22, 1575-1603.

Le Van, C. and L. Morhaim, 2002, Optimal Growth Models with Bounded or Unbounded Returns: A Unifying Approach, Journal of Economic Theory $105,158-187$.

Ladron de Guevara, A., S. Ortigueira, and M.S. Santos, 1999, A Two-Sector Model of Endogenous Growth with Leisure, Review of Economic Studies 66, 609-631.

McKenzie, L.W., 1976, Turnpike Theory, Econometrica 44, 841-865.

Negishi, T., 1960, Welfare Economics and Existence of an Equilibrium for a Competitive Economy, Metroeconomica 12, 92-97.

Stokey, N.L. and S. Rebelo, 1995, Growth-Effects of Flat-Rate Taxes, Journal of Political Economy 103, 519-550.

Yano, M., 1998, On the Dual Stability of a von Neumann Facet and the Inefficacy of Temporary Fiscal Policy, Econometrica 66 (1998), 427-451. 